Research Article

\title{
A Linear Map Acts as a Leonard Pair with Each of the Generators of $U\left(s l_{2}\right)$
}

\author{
Hasan Alnajjar (D) \\ Department of Mathematics, The University of Jordan, Amman 11942, Jordan \\ Correspondence should be addressed to Hasan Alnajjar; h.najjar@ju.edu.jo
}

Received 27 May 2020; Accepted 8 July 2020; Published 25 August 2020

Academic Editor: Luca Vitagliano

Copyright (c) 2020 Hasan Alnajjar. This is an open access article distributed under the Creative Commons Attribution License, which permits unrestricted use, distribution, and reproduction in any medium, provided the original work is properly cited.

Let $\mathscr{F}$ denote an algebraically closed field with a characteristic not two. Fix an integer $d \geq 3$; let $x, y$, and $z$ be the equitable basis of $s l_{2}$ over $\mathscr{F}$. Let $V$ denote an irreducible $s l_{2}$-module with dimension $d+1$; let $A \in \operatorname{End}(V)$. In this paper, we show that if each of the pairs $A, x, A, y$, and $A, z$ acts on $V$ as a Leonard pair, then these pairs are of Krawtchouk type. Moreover, $A$ is a linear combination of $1, x, y$, and $z$.

\section{Introduction}

In this section, we recall some facts concerning Leonard pairs. Leonard pairs were introduced by P. Terwilliger [1] to extend the algebraic approach of Bannai and Ito [2] to a result of D. Leonard concerning the sequences of orthogonal polynomials with finite support, for which the dual sequence of polynomials is also a sequence of orthogonal polynomials $[3,4]$. These polynomials arise in connection with the finitedimensional representations of certain Lie algebras and quantum groups, so one expects Leonard pairs to arise as well. Leonard pairs of Krawtchouk type have been constructed from finite-dimensional irreducible $s l_{2}$-modules $[5,6]$. Fix an integer $d \geq 1$. Throughout this paper $\mathscr{F}$ shall denote an algebraically closed field with characteristic not two. Also, $V$ shall denote an $\mathscr{F}$-vector space of dimension $d+1$, and $\operatorname{Mat}_{d+1}(\mathscr{F})$ shall denote the $\mathscr{F}$-algebra of $(d+$ $1) \times(d+1)$ matrices with entries in $\mathscr{F}$ having rows and columns indexed by $1,2, \ldots, d+1$. A square matrix is said to be tridiagonal whenever every nonzero entry appears on, immediately above, or immediately below the main diagonal. A tridiagonal matrix is said to be irreducible whenever all entries immediately above and below the main diagonal are nonzero. A square matrix is said to be upper (resp. lower) bidiagonal whenever every nonzero entry appears on or immediately above (resp. below) the main diagonal.
Definition 1. Let $V$ denote a vector space over $\mathscr{F}$ with finite positive dimension. By a Leonard pair on $V$, we mean an ordered pair $A, A^{*}$, where $A: V \longrightarrow V$ and $A^{*}: V \longrightarrow V$ are linear transformations that satisfy both (i) and (ii) below:

(i) There exists a basis for $V$ with respect to which the matrix representing $A^{*}$ is diagonal and the matrix representing $A$ is irreducible tridiagonal

(ii) There exists a basis for $V$ with respect to which the matrix representing $A$ is diagonal and the matrix representing $A^{*}$ is irreducible tridiagonal

We remark that if $A, A^{*}$ is a Leonard pair on $V$, then $A^{*}$, $A$ is a Leonard pair on $V$, and for any scalars $\alpha, \alpha^{*}$, the pair $A+\alpha I, A^{*}+\alpha^{*} I$ is also a Leonard pair on $V$.

For more details about Leonard pairs, see [7-14].

Definition 2. Let $V$ denote a vector space over $\mathscr{F}$ with dimension $d+1$. let $\left\{v_{i}\right\}_{i=0}^{d}$ denote a basis for $V$, which satisfies condition (ii) of Definition 1. For $0 \leq i \leq d$, the vector $v_{i}$ is an eigenvector of $A$; let $\theta_{i}$ denote the corresponding eigenvalues. Let $\left\{v_{i}^{*}\right\}_{i=0}^{d}$ denote a basis for $V$, which satisfies condition (i) of Definition 1 . For $0 \leq i \leq d$, the vector $v_{i}^{*}$ is an eigenvector of $A^{*}$; let $\theta_{i}^{*}$ denote the corresponding eigenvalues. Let the sequence $\left\{a_{i}\right\}_{i=0}^{d}$ denote the diagonal of the matrix, which represents $A$ with respect to $\left\{v_{i}^{*}\right\}_{i=0}^{d}$. Let the 
sequence $\left\{a_{i}^{*}\right\}_{i=0}^{d}$ denote the diagonal of the matrix, which represents $A^{*}$ with respect to $\left\{v_{i}\right\}_{i=0}^{d}$.

The ordering of $\left\{\theta_{i}\right\}_{i=0}^{d}$ in Definition 2 is said to be standard. For a standard ordering $\left\{\theta_{i}\right\}_{i=0}^{d}$ of eigenvalues of, the ordering $\left\{\theta_{d-i}\right\}_{i=0}^{d}$ is also standard and no further ordering is standard. A similar result applies for $\left\{\theta_{i}^{*}\right\}_{i=0}^{d}$.

Theorem 1 (see [15]). Let $A, A^{*}$ be a Leonard pair on $V$; let $\left\{\theta_{i}\right\}_{i=0}^{d}\left(\right.$ resp. $\left.\left(\left\{\theta_{i}^{*}\right\}_{i=0}^{d}\right)\right)$ be standard ordering of the eigenvalues of $A$ (resp. $\left.A^{*}\right)$. Then, there exists a basis of $V$ such that the matrices representing $A, A^{*}$ with respect to this basis are, respectively,

$$
\begin{aligned}
A & =\left(\begin{array}{ccccc}
\theta_{0} & & & & \\
1 & \theta_{1} & & & \\
& 1 & \theta_{2} & & \\
& & \ddots & \ddots & \\
& & & 1 & \theta_{d}
\end{array}\right), \\
A^{*} & =\left(\begin{array}{ccccc}
\theta_{0}^{*} & \varphi_{1} & & & \\
& \theta_{1}^{*} & \varphi_{2} & & \\
& & \theta_{2}^{*} & \ddots & \\
& & & \ddots & \varphi_{d} \\
& & & & \theta_{d}^{*}
\end{array}\right),
\end{aligned}
$$

for some sequence of scalars $\varphi_{1}, \varphi_{2}, \ldots, \varphi_{d}$ in $\mathscr{F}$, which we refer to as the first split sequence of $A, A^{*}$.

Definition 3 (see [16]). Let $d$ denote a nonnegative integer. By a parameter array over $\mathscr{F}$ of diameter $d$, we mean a sequence of scalars $\left(\left\{\theta_{i}\right\}_{i=0}^{d},\left\{\theta_{i}^{*}\right\}_{i=0}^{d} ;\left\{\varphi_{j}\right\}_{j=1}^{d},\left\{\phi_{j}\right\}_{j=1}^{d}\right)$ taken from $\mathscr{F}$ that satisfy the following conditions:

$$
\begin{gathered}
\theta_{i} \neq \theta_{j}, \quad(0 \leq i<j \leq d), \\
\theta_{i}^{*} \neq \theta_{j}^{*}, \quad(0 \leq i<j \leq d), \\
\varphi_{i} \neq 0, \quad(1 \leq i \leq d), \\
\phi_{i} \neq 0, \quad(1 \leq i \leq d), \\
\varphi_{i}=\phi_{1} \sum_{h=0}^{i-1} \frac{\theta_{h}-\theta_{d-h}}{\theta_{0}-\theta_{d}}+\left(\theta_{i}^{*}-\theta_{0}^{*}\right)\left(\theta_{i-1}-\theta_{d}\right), \quad(1 \leq i \leq d), \\
\phi_{i}=\varphi_{1} \sum_{h=0}^{i-1} \frac{\theta_{h}-\theta_{d-h}}{\theta_{0}-\theta_{d}}+\left(\theta_{i}^{*}-\theta_{0}^{*}\right)\left(\theta_{d-i+1}-\theta_{0}\right), \quad(1 \leq i \leq d), \\
\frac{\theta_{i-2}-\theta_{i+1}}{\theta_{i-1}-\theta_{i}}=\frac{\theta_{j-2}^{*}-\theta_{j+1}^{*}}{\theta_{j-1}^{*}-\theta_{j}^{*}}, \quad(2 \leq i, j \leq d-1) .
\end{gathered}
$$

Theorem 2 (see [15]). Let d denote a nonnegative integer; let $B$ and $B^{*}$ denote matrices in $\operatorname{Mat}_{d+1}(\mathscr{F})$. Assume $B$ is lower bidiagonal and $B^{*}$ is upper bidiagonal. Then, the following are equivalent:

(i) The pair B, $B^{*}$ is a Leonard pair in $\operatorname{Mat}_{d+1}(\mathscr{F})$.

(ii) There exists a parameter array $\left(\left\{\theta_{i}\right\}_{i=0}^{d}, \quad\left\{\theta_{i}^{*}\right\}_{i=0}^{d}\right.$; $\left\{\varphi_{j}\right\}_{j=1}^{d},\left\{\phi_{j}\right\}_{j=1}^{d}$ ) over $\mathscr{F}$ such that

$$
\begin{aligned}
B(i, i) & =\theta_{i}, B^{*}(i, i)=\theta_{i}^{*}, \quad(0 \leq i \leq d), \\
B(j, j-1) B^{*}(j-1, j) & =\varphi_{j}, \quad(1 \leq j \leq d) .
\end{aligned}
$$

Suppose ( $i)$ and (ii) hold. Then, the parameter array in (ii) is uniquely determined by $B, B^{*}$.

Theorem 3 (see [15]). With reference to Definition 2, let $A$, $A^{*}$ be a Leonard pair on $V$; let $\left\{\theta_{i}\right\}_{i=0}^{d}$ (resp. $\left.\left(\left\{\theta_{i}^{*}\right\}_{i=0}^{d}\right)\right)$ be standard ordering of the eigenvalues of $A$ (resp. $\left.A^{*}\right)$. Via a standard basis, the matrix representing $A$ and $A^{*}$ are

$$
\left(\begin{array}{cccccc}
a_{0} & b_{0} & & & & 0 \\
c_{1} & a_{1} & b_{1} & & & \\
0 & c_{2} & a_{2} & b_{2} & & \\
& \ddots & \ddots & \ddots & \ddots & \\
& & 0 & c_{d-1} & a_{d-1} & b_{d-1} \\
0 & & & 0 & c_{d} & a_{d}
\end{array}\right)
$$

and $\operatorname{diag}\left(\theta_{0}^{*}, \theta_{1}^{*}, \ldots, \theta_{d}^{*}\right)$, where

$$
\begin{aligned}
& a_{i}=\theta_{i}+\frac{\varphi_{i}}{\theta_{i}^{*}-\theta_{i-1}^{*}}+\frac{\varphi_{i+1}}{\theta_{i}^{*}-\theta_{i+1}^{*}}, \quad(0 \leq i \leq d), \\
& a_{i}^{*}=\theta_{i}^{*}+\frac{\varphi_{i}}{\theta_{i}-\theta_{i-1}}+\frac{\varphi_{i+1}}{\theta_{i}-\theta_{i+1}}, \quad(0 \leq i \leq d), \\
& b_{i}=\varphi_{i+1} \frac{\prod_{h=0}^{i-1}\left(\theta_{i}^{*}-\theta_{h}^{*}\right)}{\prod_{h=0}^{i}\left(\theta_{i+1}^{*}-\theta_{h}^{*}\right)}, \quad(0 \leq i \leq d-1), \\
& c_{i}=\phi_{i} \frac{\prod_{h=0}^{d-i-1}\left(\theta_{i}^{*}-\theta_{d-h}^{*}\right)}{\prod_{h=0}^{d-i}\left(\theta_{i-1}^{*}-\theta_{d-h}^{*}\right)}, \quad(1 \leq i \leq d),
\end{aligned}
$$

where $\varphi_{0}=0$ and $\varphi_{d+1}=0$.

Theorem 4 (see [17]). Let $V$ denote a vector space over $\mathscr{F}$ with finite positive dimension. Let $A, A^{*}$ denote a Leonard pair on $V$. Then, there exists a sequence of scalars $\beta, \gamma, \gamma^{*}, \varrho$, $\rho^{*}, \omega, \eta$, and $\eta^{*}$ taken from $\mathscr{F}$ such that

$$
\begin{aligned}
& A^{2} A^{*}-\beta A A^{*} A+A^{*} A^{2}-\gamma\left(A A^{*}+A^{*} A\right) \\
&-\varrho A^{*}=\gamma^{*} A^{2}+\omega A+\eta I, \\
& A^{*^{2}} A-\beta A^{*} A A^{*}+A A^{*^{2}}-\gamma^{*}\left(A^{*} A+A A^{*}\right) \\
&-\varrho^{*} A=\gamma A^{*^{2}}+\omega A^{*}+\eta^{*} I .
\end{aligned}
$$


The sequence is uniquely determined by the pair $A, A^{*}$ provided the dimension of $V$ is at least 4.

Relations (12) and (13) are called the Askey-Wilson relations.

\section{Leonard Pairs of Classical Type}

In [18], the parameters arrays are classified into 13 families, each named for certain associated sequences of orthogonal polynomials. The four families, which arise in this paper, share certain property. Given a parameter array, let $\beta$ be the common value of (8) minus one.

Definition 4. A parameter array is of classical type whenever $\beta=2$.

Theorem 5 (see [18]). A parameter array is of classical type if and only if it is of Racah, Hahn, dual Hahn, or Krawtchouk type.

Theorem 6 (see [18], Example 5.10). Fix nonzero $h, h^{*} \in \mathscr{F}$ and $s, s^{*}, r_{1}, r_{2}, \theta_{0}, \theta_{0}^{*} \in \mathscr{F}$ such that $r_{1}+r_{2}=s+s^{*}+d+1$ and none of $r_{1}, r_{2}, s^{*}-r_{1}, s^{*}-r_{2}$ is equal to $-i$ for $1 \leq i \leq d$ and that neither of $s, s^{*}$ is equal to $-i$ for $2 \leq i \leq 2 d$. Let

$$
\begin{aligned}
\theta_{i} & =\theta_{0}+h i(i+1+s), \quad(0 \leq i \leq d), \\
\theta_{i}^{*} & =\theta_{0}^{*}+h^{*} i\left(i+1+s^{*}\right), \quad(0 \leq i \leq d), \\
\varphi_{j} & =h h^{*} j(j-d-1)\left(j+r_{1}\right)\left(j+r_{2}\right), \quad(1 \leq j \leq d), \\
\phi_{j} & =h h^{*} j(j-d-1)\left(j+s^{*}-r_{1}\right)\left(j+s^{*}-r_{2}\right), \quad(1 \leq j \leq d) .
\end{aligned}
$$

Then, $\Phi=\left(\left\{\theta_{i}\right\}_{i=0}^{d},\left\{\theta_{i}^{*}\right\}_{i=0}^{d} ;\left\{\varphi_{j}\right\}_{j=1}^{d},\left\{\phi_{j}\right\}_{j=1}^{d}\right)$ is a parameter array of Racah type. We refer to the scalars $r_{1}, r_{2}, s, s^{*}, h, h^{*}$, $\theta_{0}$, and $\theta_{0}^{*}$ as hypergeometric parameters of $\Phi$.

Theorem 7 (see [18], Example 5.11). Fix nonzero $s, h^{*} \in \mathscr{F}$ and some $s^{*}, r, \theta_{0}, \theta_{0}^{*} \in \mathscr{F}$ such that neither of $r, s^{*}-r$ is equal to $-i$ for $1 \leq i \leq d$ and that $s^{*}$ is not equal $-i$ for $2 \leq i \leq 2 d$. Let

$$
\begin{aligned}
\theta_{i} & =\theta_{0}+s i, \quad(0 \leq i \leq d), \\
\theta_{i}^{*} & =\theta_{0}^{*}+h^{*} i\left(i+1+s^{*}\right), \quad(0 \leq i \leq d), \\
\varphi_{j} & =h^{*} s j(j-d-1)(j+r), \quad(1 \leq j \leq d), \\
\phi_{j} & =-h^{*} s j(j-d-1)\left(j+s^{*}-r\right), \quad(1 \leq j \leq d) .
\end{aligned}
$$

Then, $\Phi=\left(\left\{\theta_{i}\right\}_{i=0}^{d},\left\{\theta_{i}^{*}\right\}_{i=0}^{d} ;\left\{\varphi_{j}\right\}_{j=1}^{d},\left\{\phi_{j}\right\}_{j=1}^{d}\right)$ is a parameter array of Hahn type. We refer to the scalars $r, s, s^{*}$, $h^{*}, \theta_{0}$, and $\theta_{0}^{*}$ as hypergeometric parameters of $\Phi$.

Theorem 8 (see [18], Example 5.12). Fix nonzero $h, s^{*} \in \mathscr{F}$ and $s, r, \theta_{0}, \theta_{0}^{*} \in \mathscr{F}$ such that neither of $r, s-r$ is equal to $-i$ for $1 \leq i \leq d$, and that $s$ is not equal $-i$ for $2 \leq i \leq 2 d$. Let

$$
\begin{aligned}
\theta_{i} & =\theta_{0}+h i(i+1+s), \quad(0 \leq i \leq d), \\
\theta_{i}^{*} & =\theta_{0}^{*}+s^{*} i, \quad(0 \leq i \leq d), \\
\varphi_{j} & =h s^{*} j(j-d-1)(j+r), \quad(1 \leq j \leq d), \\
\phi_{j} & =h s^{*} j(j-d-1)(j+r-s-d-1), \quad(1 \leq j \leq d) .
\end{aligned}
$$

Then, $\Phi=\left(\left\{\theta_{i}\right\}_{i=0}^{d},\left\{\theta_{i}^{*}\right\}_{i=0}^{d} ;\left\{\varphi_{j}\right\}_{j=1}^{d},\left\{\phi_{j}\right\}_{j=1}^{d}\right)$ is a parameter array of dual Hahn type. We refer to the scalars $r, s$, $s^{*}, h, \theta_{0}$, and $\theta_{0}^{*}$ as hypergeometric parameters of $\Phi$.

Theorem 9 (see [18]. Example 5.13). Fix nonzero $r, s$, $s^{*} \in \mathscr{F}$, and some $\theta_{0}, \theta_{0}^{*} \in \mathscr{F}$ such that $r \neq s s^{*}$ :

$$
\begin{aligned}
\theta_{i} & =\theta_{0}+s i, \quad(0 \leq i \leq d), \\
\theta_{i}^{*} & =\theta_{0}^{*}+s^{*} i, \quad(0 \leq i \leq d), \\
\varphi_{j} & =r j(j-d-1), \quad(1 \leq j \leq d), \\
\phi_{j} & =\left(r-s s^{*}\right) j(j-d-1), \quad(1 \leq j \leq d) .
\end{aligned}
$$

Then, $\Phi=\left(\left\{\theta_{i}\right\}_{i=0}^{d},\left\{\theta_{i}^{*}\right\}_{i=0}^{d} ;\left\{\varphi_{j}\right\}_{j=1}^{d},\left\{\phi_{j}\right\}_{j=1}^{d}\right)$ is a parameter array of Krawtchouk type. We refer to the scalars $r$, $s, s^{*}, \theta_{0}$, and $\theta_{0}^{*}$ as hypergeometric parameters of $\Phi$.

\section{The Lie Algebra $s l_{2}$}

In this section, we recall some facts concerning the Lie algebra $s l_{2}$.

Definition 5 (see [11]). The Lie algebra $s l_{2}$ is the $\mathscr{F}$-algebra that has a basis $e, f, h$ satisfying the following conditions:

$$
\begin{aligned}
{[h, e] } & =2 e, \\
{[h, f] } & =-2 f, \\
{[e, f] } & =h,
\end{aligned}
$$

where $[-,-]$ denotes the Lie bracket.

Lemma 1 (see [10]). With reference to Definition 5, let

$$
\begin{aligned}
& x=2 e-h, \\
& y=-2 f-h, \\
& z=h .
\end{aligned}
$$

Then, $x, y, z$ is a basis for $s l_{2}$ and

$$
\begin{aligned}
& {[x, y]=2 x+2 y,} \\
& {[y, z]=2 y+2 z,} \\
& {[z, x]=2 z+2 x .}
\end{aligned}
$$

We call $x, y, z$ the equitable basis for the Lie algebra $s l_{2}$.

Lemma 2 (see [11]). For each nonnegative integer $d$, there is an irreducible finite-dimensional sl $l_{2}$-module $V_{d}$ with basis $v_{0}, v_{1}, \ldots, v_{d}$ and action $h v_{i}=(d-2 i) v_{i}(0 \leq i \leq d)$, $f v_{i}=(i+1) v_{i+1}(0 \leq i \leq d-), \quad f v_{d}=0, \quad e v_{0}=0, \quad$ and ev $v_{i}=(d-i+1) v_{i-1}(1 \leq i \leq d) . \quad$ Moreover, up to 
isomorphism, $V_{d}$ is the unique irreducible $l_{2}$-module of dimension $d+1$.

Lemma 3 (see [10]). With reference to Lemmas 1 and 2,

$$
\begin{aligned}
(x+d I) v_{0} & =0 \\
(x+(d-2 i) I) v_{i} & =2(d-i+1) v_{i-1}, \quad(1 \leq i \leq d), \\
(y+(d-2 i) I) v_{i} & =-2(i+1) v_{i+1}, \quad(0 \leq i \leq d-1), \\
(y-d I) v_{d} & =0 \\
z v_{i} & =(d-2 i) v_{i}, \quad(0 \leq i \leq d) .
\end{aligned}
$$

Definition 6. With reference to Lemmas 1 and 2 , fix $\zeta \in \mathfrak{C}$. A basis $b=\left\{b_{0}, b_{1}, \ldots, b_{d}\right\}$ of $V_{d}$ is said to be a standard $\zeta(z)$-eigenbasis whenever $\zeta(x), \zeta(y)$, and $\zeta(z)$ act as

$$
\begin{aligned}
(\zeta(x)+d I) b_{0} & =0 \\
(\zeta(x)+(d-2 i) I) b_{i} & =2(d-i+1) b_{i-1}, \quad(1 \leq i \leq d) \\
(\zeta(y)+(d-2 i) I) b_{i} & =-2(i+1) b_{i+1}, \quad(0 \leq i \leq d-1) \\
(\zeta(y)-d I) b_{d} & =0, \\
(\zeta(z)-(d-2 i) I) b_{i} & =0, \quad(0 \leq i \leq d) .
\end{aligned}
$$

Lemma 4 (see [19]). With reference to Lemmas 1 and 2,

(i) For $0 \leq i \leq d$, let $u_{i}=\sum_{j=0}^{d-i}(-1)^{i}((d-j) ! / i !(d-$ $j-i) !) v_{j}$. Then, $u=\left\{u_{0}, u_{1}, \ldots, u_{d}\right\}$ is a standard $x$-eigenbasis of $V_{d}$.

(ii) For $0 \leq i \leq d$, let $w_{i}=\sum_{j=d-i}^{d}(-1)^{i}(j ! /(d-i) !(j+$ $i-d) !) v_{j}$. Then, $w=\left\{w_{0}, w_{1}, \ldots, w_{d}\right\}$ is a standard $y$-eigenbasis of $V_{d}$.

(iii) Let $v_{0}, v_{1}, \ldots, v_{d}$ be as in Lemma 2. Then, $v=\left\{v_{0}, v_{1}, \ldots, v_{d}\right\}$ is a standard $z$-eigenbasis of $V_{d}$ [10].

The main results of this paper are the following theorems.

Theorem 10. Fix an integer. $d \geq 3$; let $x, y$, and $z$ be the equitable basis of $s l_{2}$ over $\mathscr{F}$. Let $V$ denote an irreducible $s l_{2}$-module with dimension $d+1$; let $A \in \operatorname{End}(V)$. Then, the following are equivalent:

(i) Any two of the pairs A, x, A, y, and A, z are Leonard pairs

(ii) All the pairs A, x, A, y, and A,z are Leonard pairs

Theorem 11. Fix an integer $d \geq 3$; let $x, y$, and $z$ be the equitable basis of $s l_{2}$ over $\mathscr{F}$. Let $V$ denote an irreducible $s_{2}$-module with dimension $d+1$; let $A \in \operatorname{End}(V)$ such that each of the pairs $A, x, A, y$, and $A, z$ is a Leonard pair; then, all the pairs are of Krawtchouk type. Moreover, $A$ is a linear combination of $1, x, y$, and $z$.
We remark here that the authors in [12] proved similar result with the generators of the quantum algebra $U_{q}\left(s l_{2}\right)$.

\section{The Type of Leonard Pair}

We start recalling some facts which help us to determine the type of the Leonard pairs.

Lemma 5 (see [17]). Let d denote a nonnegative integer, and let $V$ denote a vector space over $\mathscr{F}$ with dimension $d+1$. Let $A, A^{*}$ denote a Leonard pair on $V$ with fundamental parameter $\beta$. Let the scalars $\theta_{i}$ and $\theta_{i}^{*}$ be as in Definition 2; then, there exist scalars $\gamma, \gamma^{*}, \varrho$, and $\varrho^{*}$ such that
(i) $\gamma=\theta_{i-1}-\beta \theta_{i}+\theta_{i+1}, \quad 1 \leq i \leq d-1$
(ii) $\gamma^{*}=\theta_{i-1}^{*}-\beta \theta_{i}^{*}+\theta_{i+1}^{*}, \quad 1 \leq i \leq d-1$
(iii) $\varrho=\theta_{i-1}^{2}-\beta \theta_{i-1} \theta_{i}+\theta_{i}^{2}-\gamma\left(\theta_{i-1}+\theta_{i}\right), \quad 1 \leq i \leq d$
(iv) $\varrho^{*}=\theta_{i-1}^{*^{2}}-\beta \theta_{i-1}^{*} \theta_{i}^{*}+\theta_{i}^{*^{2}}-\gamma^{*}\left(\theta_{i-1}^{*}+\theta_{i}^{*}\right), \quad 1 \leq i \leq d$

Theorem 12 (see [17]). Let d denote a nonnegative integer, and let $V$ denote a vector space over $\mathscr{F}$ with dimension $d+1$. Let $A, A^{*}$ denote a Leonard pair on $V$. Let $\beta, \gamma, \gamma^{*}, \varrho, \rho^{*}, \omega, \eta$, and $\eta^{*}$ denote a sequence of scalars taken from $\mathscr{F}$, which satisfy (12) and (13). Let the scalars $\theta_{i}, \theta_{i}^{*}, a_{i}$, and $a_{i}^{*}$ be as in Definition 2. Then, the following hold:

(i) $\omega=a_{i}^{*}\left(\theta_{i}-\theta_{i+1}\right)+a_{i-1}^{*}\left(\theta_{i-1}-\theta_{i-2}\right)-\gamma^{*}\left(\theta_{i}+\theta_{i-1}\right)$, $1 \leq i \leq d$

(ii) $\omega=a_{i}\left(\theta_{i}^{*}-\theta_{i+1}^{*}\right)+a_{i-1}\left(\theta_{i-1}^{*}-\theta_{i-2}^{*}\right)-\gamma\left(\theta_{i}^{*}+\theta_{i-1}^{*}\right)$, $1 \leq i \leq d$

(iii) $\eta=a_{i}^{*}\left(\theta_{i}-\theta_{i-1}\right)\left(\theta_{i}-\theta_{i+1}\right)-\gamma^{*} \theta_{i}^{2}-\omega \theta_{i}, \quad 0 \leq i \leq d$

(iv) $\eta^{*}=a_{i}\left(\theta_{i}^{*}-\theta_{i-1}^{*}\right)\left(\theta_{i}^{*}-\theta_{i+1}^{*}\right)-\gamma \theta_{i}^{*^{2}}-\omega \theta_{i}^{*}, \quad 0 \leq i \leq d$

Lemma 6. With reference to Lemma 1 , if each of the pairs $A, x, A, y$, and $A, z$ is a Leonard pair, then all the pairs are of dual Hahn type or all the pairs of Krawtchouk type.

Proof. Note that, from Definition 4 and Lemma 3, all the pairs are of classical type. Now, the result holds by Theorem 5 and Theorem 6-Theorem 9.

We have two cases to check, the first case, is there $A$ such that all the pairs $A, x, A, y$, and $A, z$ are Leonard pairs of dual Hahn type?, and the second case, is there $A$ such that all the pairs $A, x, A, y$, and $A, z$ are Leonard pairs of Krawtchouk type? We start with the dual Hahn case.

For the rest of the paper, fix an integer $d \geq 3$; let $V_{d}$ denote an irreducible $s l_{2}$-module with dimension $d+1$; let $A \in \operatorname{End}\left(V_{d}\right)$; Let $[T]_{b}$ be the matrix that represents the linear map $T$ with respect to the basis $b$.

Lemma 7. With reference to Lemma 3 , let $\xi \in\{x, y, z\}$, assume that $A, \xi$ is a Leonard pair of dual Hahn type; let $\left(\left\{\theta_{i}\right\}_{i=0}^{d},\left\{\theta_{i}^{*}\right\}_{i=0}^{d} ;\left\{\varphi_{j}\right\}_{j=1}^{d},\left\{\phi_{j}\right\}_{j=1}^{d}\right)$ be the parameter array associated with the pair $A$, $\xi$; then, 


$$
\begin{aligned}
\theta_{i} & =\theta_{0}+h i(i+1+s), \quad 0 \leq i \leq d, \\
\theta_{i}^{*} & =\theta_{0}^{*}+h_{\xi} i, \quad 0 \leq i \leq d, \\
\varphi_{i} & =h h_{\xi} i(i-d-1)\left(i+r_{\xi}\right), \quad 1 \leq i \leq d, \\
\phi_{i} & =h h_{\xi} i(i-d-1)\left(i+r_{\xi}-s-d-1\right), \quad 1 \leq i \leq d,
\end{aligned}
$$

where $h$ and $h_{\xi}$ are nonzero scalars, none of $r_{\xi}, s-r_{\xi}$ equal to $-i$ for $1 \leq i \leq d$, and $s \neq i$ for $2 \leq i \leq 2 d$.

Proof. Clear from Theorem 8.

Lemma 8. Let $A \in \operatorname{End}\left(V_{d}\right)$ such that $A, z$ is a Leonard pair of dual Hahn type; then, the basis $\left\{e_{i}\right\}_{i=0}^{d}$ for $V_{d}$ with respect to which the matrix representing $A$ is irreducible tridiagonal and the matrix representing $z$ is diagonal is $\left\{k_{i} v_{i}\right\}_{i=0}^{d}$ or $\left\{k_{d-i} v_{d-i}\right\}_{i=0}^{d}$ for some nonzero scalars $k_{i} \in \mathscr{F}$.

Proof. Clear from Lemma 3 and the paragraph after Definition 2.

Since the case with basis $\left\{k_{d-i} v_{d-i}\right\}_{i=0}^{d}$ can be treated similar to the case with basis $\left\{k_{i} v_{i}\right\}_{i=0}^{d}$, we shall prove our results only for the case $\left\{k_{i} v_{i}\right\}_{i=0}^{d}$. Let $\left\{e_{i}=k_{i} v_{i}\right\}_{i=0}^{d}$.

Lemma 9. With reference to Lemmas 2 and 8,

$$
\begin{aligned}
(x+d I) e_{0} & =0, \\
(x+(d-2 i) I) e_{i} & =\frac{2(d-i+1) k_{i}}{k_{i-1}} e_{i-1}, \quad(1 \leq i \leq d), \\
(y+(d-2 i) I) e_{i} & =\frac{-2(i+1) k_{i}}{k_{i+1}} e_{i+1}, \quad(0 \leq i \leq d-1), \\
(y-d I) e_{d} & =0, \\
z e_{i} & =(d-2 i) e_{i}, \quad(0 \leq i \leq d) .
\end{aligned}
$$

Lemma 10. With reference to Lemmas 7 and 8 , let $A, z$ be a Leonard pair of dual Hahn type; then,

$$
\begin{aligned}
& A e_{i}=\beta_{i-1}^{*} e_{i-1}+\alpha_{i}^{*} e_{i}+\lambda_{i+1}^{*} e_{i+1}, \quad 1 \leq i \leq d-1, \\
& A e_{0}=\alpha_{0}^{*} e_{0}+\lambda_{1}^{*} e_{1}, \\
& A e_{d}=\beta_{d-1}^{*} e_{d-1}+\alpha_{d}^{*} e_{d},
\end{aligned}
$$

where

$$
\begin{aligned}
& \beta_{i}^{*}=h(d-i)\left(i+1+r_{z}\right), \quad 0 \leq i \leq d-1, \\
& \alpha_{i}^{*}=\theta_{0}+h\left(i s+(d-2 i) r_{z}+2 i(d-i)+d\right), \quad 0 \leq i \leq d, \\
& \lambda_{i}^{*}=h i\left(i-1-d+r_{z}+s\right), \quad 1 \leq i \leq d .
\end{aligned}
$$

Proof. The result is held by Theorem 3 and Lemmas 7 and 9.
Lemma 11. With reference to Lemma 5 and Theorem 12, let $\xi \in\{x, y, z\}$; assume $A, \xi$ is a Leonard pair of dual Hahn type; let $\theta_{i}=0$ and $\theta_{i}^{*}=0$; then,

$$
\begin{aligned}
\gamma & =2 h, \\
\gamma^{*} & =0, \\
\varrho & =s h^{2}(2+s), \\
\varrho^{*} & =h_{\xi}^{2}, \\
\omega & =h h_{\xi}\left(2 r_{\xi}-s-2 d\right), \\
\eta & =-d h^{2} h_{\xi} s\left(1+r_{\xi}\right), \\
\eta^{*} & =-d h h_{\xi}^{2}\left(1+r_{\xi}\right) .
\end{aligned}
$$

Proof. Clear from Lemma 7.

Lemma 12. Let $A \in \operatorname{End}\left(V_{d}\right)$; assume $A, z$ is a Leonard pair of dual Hahn type; then, the pair A, $y$ is not a Leonard pair of dual Hahn type.

Proof. Let

$$
\begin{aligned}
H= & A^{*^{2}} A-\beta A^{*} A A^{*}+A A^{*^{2}}-\gamma^{*}\left(A^{*} A+A A^{*}\right) \\
& -\varrho^{*} A-\gamma A^{*^{2}}+\omega A^{*}+\eta^{*} I .
\end{aligned}
$$

Since the pair $A, z$ is a Leonard pair, the action of $A$ on the basis $e=\left\{e_{i}\right\}_{i=0}^{d}$ is given in Lemma 10, and the action of $y$ is given in Lemma 9. Assume that the pair $A, y$ is a Leonard pair and let $y=A^{*}$; hence, by Theorem 4 , there exists a sequence of scalars $\beta, \gamma, \gamma^{*}, \varrho, \rho^{*}, \omega, \eta$, and $\eta^{*}$ as in Lemma 11 such that $[H]_{e}=0$. Now, if we solve $[H]_{e}[1,1]$ for $r_{y}$, we find that $r_{y}=k_{1} r_{z}-k_{0}\left(r_{z}+1\right) / k_{1}$. Substitute $r_{y}$; then, for $1 \leq i \leq d-2$, solve the $(i+1, i)$-entries of $[H]_{e}$ for $k_{i}$; we find that $k_{i}=k_{i+1}\left(r_{z}+i+2\right) / k_{i+2}\left(r_{z}+i+1\right)$. Substitute $k_{i}$ to find that

$$
[H]_{e}[4,1]=\frac{48 k_{d-1}^{2} h\left(r_{z}+d\right)^{2}}{k_{d}\left(r_{z}+1\right)\left(r_{z}+2\right)\left(r_{z}+3\right)} .
$$

By Lemmas 7 and $8, h \neq 0, r_{z}+d \neq 0$, and $k_{d-1} \neq 0$, so $[H]_{e}[4,1] \neq 0$. Hence, the pair $A, y$ is not a Leonard pair.

Lemma 13. Let $A, z$ be a Leonard pair of dual Hahn type; then, the pair A, $x$ is not a Leonard pair of dual Hahn type.

Proof. Similar to proof of Lemma 12.

\section{Leonard Pair of Krawtchouk Type}

In this section, we describe a linear map $A$ such that all the pairs $A, x, A, y$, and $A, z$ are Leonard pairs of Krawchouch type.

Lemma 14. With reference to Lemma 3, let $\xi \in\{x, y, z\}$, assume that $A, \xi$ is a Leonard pair of Krawtchouk type; let $\left(\left\{\theta_{i}\right\}_{i=0}^{d}, \quad\left\{\theta_{i}^{*}\right\}_{i=0}^{d} ;\left\{\varphi_{j}\right\}_{j=1}^{d},\left\{\phi_{j}\right\}_{j=1}^{d}\right)$ be the parameter array 
associated with the pair $A, \xi$; then, there exist a nonzero scalars $h, h_{\xi}$, and $r_{\xi}$ such that $r_{\xi}-h h_{\xi} \neq 0$, and

$$
\begin{aligned}
\theta_{i} & =\theta_{0}+h i, \quad 0 \leq i \leq d, \\
\theta_{i}^{*} & =\theta_{0}^{*}+h_{\xi} i, \quad 0 \leq i \leq d, \\
\varphi_{i} & =r_{\xi} i(i-d-1), \quad 1 \leq i \leq d, \\
\phi_{i} & =\left(r_{\xi}-h h_{\xi}\right) i(i-d-1), \quad 1 \leq i \leq d,
\end{aligned}
$$

Proof. Clear from Theorem 9.

Lemma 15. With reference to Lemma 5 and Theorem 12, assume $A, \xi$ is a Leonard pair of Krawtchouk type; let $\theta_{i}=0$ and $\theta_{i}^{*}=0$; then,

$$
\begin{aligned}
\gamma & =0, \\
\gamma^{*} & =0, \\
\varrho & =h^{2}, \\
\varrho^{*} & =h_{\xi}, \\
\omega & =-\left(h h_{\xi}-2 r_{\xi}\right), \\
\eta & =-d h r_{\xi}, \\
\eta^{*} & =-d h_{\xi} r_{\xi} .
\end{aligned}
$$

Proof. Clear from Lemma 14.

Lemma 16. With reference to Lemmas 14 and 8 , Let $A, z$ be a Leonard pair of Krawtchouk type; then, $A e_{i}=\beta_{i-1} e_{i-1}+\alpha_{i} e_{i}+$ $\lambda_{i+1} e_{i+1} 1 \leq i \leq d-1, A e_{0}=\alpha_{0} e_{0}+\lambda_{1} e_{1}$, and $A e_{d}=\beta_{d-1} e_{d-1}+$ $\alpha_{d} e_{d}$, where

$$
\begin{aligned}
& \beta_{i}=\frac{(d-i) r_{z}}{2}, \quad 0 \leq i \leq d-1, \\
& \alpha_{i}=\theta_{0}+i h-\frac{(d-2 i) r_{z}}{2}, \quad 0 \leq i \leq d, \\
& \lambda_{i}=\frac{-i\left(r_{z}+2 h\right)}{2}, \quad 1 \leq i \leq d .
\end{aligned}
$$

Proof. The result holds by Theorem 3 and Lemmas 3 and 14.

Lemma 17. With reference to Lemma 16, the pair $[A]_{e},[z]_{e}$ is a Leonard pair of Krawtchouk type in $\operatorname{Mat}_{d+1}(\mathscr{F})$ if and only if $h \neq 0, r_{z} \neq 0$, and $r_{z}+2 h \neq 0$.

Proof. Let $P$ be $(d+1) \times(d+1)$ matrix indexed $1,2, \ldots, d+1$ such that the $(i, j)$-entry is

$$
P_{i j}= \begin{cases}\frac{(-2)^{j-1}(i-1) !}{(i-j) !}, & j \leq i, \\ 0, & j>i .\end{cases}
$$

Then, the matrices represent $P^{-1}[A]_{e} P$ and $P^{-1}[z]_{e} P$ as in (1), where $\left\{\theta_{i}\right\}_{i=0}^{d},\left\{\theta_{i}^{*}\right\}_{i=0}^{d} ;\left\{\varphi_{i}\right\}_{i=1}^{d}$ and $\left\{\phi_{i}\right\}_{i=1}^{d}$ are as in Lemma 14 . Hence, by Theorem $2, P^{-1}[A]_{e} P, P^{-1}[z]_{e} P$ is a Leonard pair of Krawtchouk type if and only if $h \neq 0, r_{z} \neq 0$, and $r_{z}+2 h \neq 0$ hold, which implies that the pair $[A]_{e},[z]_{e}$ is a Leonard pair of Krawtchouk type if and only if $h \neq 0, r_{z} \neq 0$, and $r_{z}+2 h \neq 0$.

Lemma 18. With reference to Lemmas 8 and 14, assume $[A]_{e},[z]_{e}$ is a Leonard pair of Krawtchouk type. If the pair $[A]_{e},[y]_{e}$ is a Leonard pair, then there exists a nonzero scalar $t \in \mathscr{F}$ such that $k_{i} / k_{i-1}=t(1 \leq i \leq d)$. Moreover, $r_{y}=r_{z}(1-t) / t$.

Proof. Routine calculations using Theorem 4 and Lemma 15.

Lemma 19. With reference to Lemmas 8 and 14, assume $[A]_{e},[z]_{e}$ is a Leonard pair of Krawtchouk type. If the pair $[A]_{e},[x]_{e}$ is a Leonard pair, then there exists a nonzero scalar $t \in \mathscr{F}$ such that $k_{i} / k_{i-1}=t(1 \leq i \leq d)$. Moreover, $r_{x}=t\left(r_{z}+2 h\right)-r_{z}$

Proof. Routine calculations using Theorem 4 and Lemma 15.

Lemma 20. Suppose that the pairs $A, z$ and $A, y$ are Leonard pairs of Krawtchouk type; let $\left\{\theta_{i}\right\}_{i=0}^{d}, \quad\left\{\theta_{i}^{*}\right\}_{i=0}^{d}$ and $\left\{\varphi_{i}\right\}_{i=1}^{d}$, $\left\{\phi_{i}\right\}_{i=1}^{d}$ be the parameter array associated with the pair $A, y$. Then, there exist a nonzero scalars $h, r_{z}$, and $t$ such that $t \neq 1$, $r_{z}+2 h \neq 0, r_{z}-t\left(r_{z}+2 h\right) \neq 0$, and

$$
\begin{aligned}
\theta_{i} & =\theta_{0}+h i, \quad 0 \leq i \leq d, \\
\theta_{i}^{*} & =2 i-d, \quad 0 \leq i \leq d, \\
\varphi_{i} & =\frac{r_{z}(1-t) i(i-d-1)}{t}, \quad 1 \leq i \leq d, \\
\phi_{i} & =\frac{\left(r_{z}-t\left(r_{z}+2 h\right)\right) i(i-d-1)}{t}, \quad 1 \leq i \leq d .
\end{aligned}
$$

Proof. Let $k_{0}=1$ in Lemma 8 , so the basis $e=\left\{t^{i} v_{i}\right\}_{i=0}^{d}$. Hence, the result holds by Lemmas 14 and 18 .

Lemma 21. Suppose that the pairs $A, z$ and $A, x$ are Leonard pairs of Krawtchouk type, let $\left\{\theta_{i}\right\}_{i=0}^{d}, \quad\left\{\theta_{i}^{*}\right\}_{i=0}^{d}$ and $\left\{\varphi_{i}\right\}_{i=1}^{d}$, $\left\{\phi_{i}\right\}_{i=1}^{d}$ be the parameter array associated with the pair $A, x$. Then, there exist nonzero scalars $h, r_{z}$, and $t$ such that $t \neq 1$, $r_{z}+2 h \neq 0, r_{z}-t\left(r_{z}+2 h\right) \neq 0$, and

$$
\begin{aligned}
\theta_{i} & =\theta_{0}+h i, \quad 0 \leq i \leq d, \\
\theta_{i}^{*} & =2 i-d, \quad 0 \leq i \leq d, \\
\varphi_{i} & =\left(t\left(r_{z}+2 h\right)-r_{z}\right) i(i-d-1), \quad 1 \leq i \leq d, \\
\phi_{i} & =\left((t-1)\left(r_{z}+2 h\right)\right) i(i-d-1), \quad 1 \leq i \leq d .
\end{aligned}
$$


Proof. Let $k_{0}=1$ in Lemma 8, so the basis $e=\left\{t^{i} v_{i}\right\}_{i=0}^{d}$. Hence, the result holds by Lemmas 14 and 19 .

Lemma 22. With reference to Lemma 2, let $t \in \mathscr{F}$ be a nonzero scalar; let $e=\left\{t^{i} v_{i}\right\}_{i=0}^{d}$. Let $A$ be as in Lemma 16; assume that $[A]_{e},[z]_{e}$ is a Leonard pair of Krawtchouk type; then, the pair $[A]_{e},[y]_{e}$ is a Leonard pair of Krawtchouk type if and only if $t \neq 1$ and $r_{z}-t\left(r_{z}+2 h\right) \neq 0$.

Proof. The pair $[A]_{e},[z]_{e}$ is a Leonard pair of Krawtchouk type; then, by Lemma $17, h \neq 0, r_{z} \neq 0$, and $r_{z}+2 h \neq 0$.

Let $P$ be $(d+1) \times(d+1)$ matrix indexed $1,2, \ldots, d+1$ such that the $(i, j)$-entry is

$$
P_{i j}= \begin{cases}\frac{(2)^{j-1}(t-1)^{j-1}(i-1) !}{t^{j-1}(i-j) !}, & j \leq i, \\ 0, & j>i .\end{cases}
$$

Then, the matrices represent $P^{-1}[A]_{e} P, P^{-1}[y]_{e} P$ as in (1), where $\left\{\theta_{i}\right\}_{i=0}^{d},\left\{\theta_{i}^{*}\right\}_{i=0}^{d}$ and $\left\{\varphi_{i}\right\}_{i=1}^{d},\left\{\phi_{i}\right\}_{i=1}^{d}$ are as in Lemma 20. Hence, by Theorem $2, P^{-1}[A]_{e} P, P^{-1}[y]_{e} P$ is a Leonard pair of Krawtchouk type, if and only if $t \neq 1$ and $r_{z}-t\left(r_{z}+\right.$ $2 h) \neq 0$ hold, which implies that the pair $[A]_{e},[y]_{e}$ is a Leonard pair of Krawtchouk type if and only if $t \neq 1$ and $r_{z}-t\left(r_{z}+2 h\right) \neq 0$.

Lemma 23. With reference to Lemma 2, let $t \in \mathscr{F}$ be a nonzero scalar; let $e=\left\{t^{i} v_{i}\right\}_{i=0}^{d}$. Let $A$ be as in Lemma 16; assume, that $[A]_{e},[z]_{e}$ is a Leonard pair of Krawtchouk type; then, the pair $[A]_{e},[x]_{e}$ is a Leonard pair of Krawtchouk type if and only if $t \neq 1$ and $r_{z}-t\left(r_{z}+2 h\right) \neq 0$.

Proof. The pair $[A]_{e},[z]_{e}$ is a Leonard pair of Krawtchouk type; then, by Lemma $17, h \neq 0, r_{z} \neq 0$, and $r_{z}+2 h \neq 0$.

Let $P$ be $(d+1) \times(d+1)$ matrix indexed $1,2, \ldots, d+1$ such that the $(i, j)$-entry is

$$
P_{i j}= \begin{cases}\frac{(2)^{j-d-1}(t-1)^{j-1}(d-i+1) !}{t^{d} d !(d-i-j+) !}, & j \leq d-i+2, \\ 0, & j>d-i+2 .\end{cases}
$$

Then,

$$
\begin{aligned}
P^{-1}[x]_{e} P & =\left(\begin{array}{cccccc}
\theta_{d} & & & & & \\
1 & \theta_{d-1} & & & \\
& 1 & \theta_{d-2} & & \\
& & \ddots & \ddots & \\
& & & & 1 & \theta_{0}
\end{array}\right), \\
P^{-1}[A]_{e} P & =\left(\begin{array}{ccccc}
\theta_{0}^{*} & \phi_{1} & & & \\
& \theta_{1}^{*} & \phi_{2} & & \\
& & \theta_{2}^{*} & \ddots & \\
& & & \ddots & \phi_{d} \\
& & & & \theta_{d}^{*}
\end{array}\right),
\end{aligned}
$$

where $\left\{\theta_{i}\right\}_{i=0}^{d},\left\{\theta_{i}^{*}\right\}_{i=0}^{d}$ and $\left\{\varphi_{i}\right\}_{i=1}^{d},\left\{\phi_{i}\right\}_{i=1}^{d}$ are as in Lemma 21 . Let $G=T^{-1} K T^{*}$, where $T, K$, and $T^{*}$ are in $\operatorname{Mat}_{d+1}(\mathscr{F})$ such that $T_{i+1, j+1}=\Pi_{h=0}^{j-1}\left(\theta_{i}^{*}-\theta_{h}^{*}\right), \quad T_{i+1, j+1}^{*}=\prod_{h=0}^{j-1}\left(\theta_{d-i}^{*}-\theta_{d-h}^{*}\right)$ for $0 \leq i, j \leq d$, and $K_{i j}=1$ if $i+j=d+2$ and 0 otherwise. Then, the matrices represent $G P^{-1}[A]_{e} P G^{-1}$, $G P^{-1}[x]_{e} P G^{-1}$ as in (1). The rest of the proof will be similar to proof of Lemma 22.

We remark here that the existence of $G$ that appears in proof of Lemma 23 was proved by Terwilliger in [18].

Lemma 24. With reference to Lemma 2, let $t \in \mathscr{F}$ be a nonzero scalar; let $e=\left\{t^{i} v_{i}\right\}_{i=0}^{d}$. Let $A$ be as in Lemma 16, assume that $[A]_{e},[z]_{e}$ is a Leonard pair of Krawtchouk type; then, $[A]_{e},[x]_{e}$ is a Leonard pair of Krawtchouk type if and only if $[A]_{e},[y]_{e}$ is a Leonard pair of Krawtchouk type.

Proof. Clear from Lemmas 22 and 23.

Lemma 25. Fix an integer $d \geq 3$, let $t \in \mathscr{F}$ be a nonzero scalar; let $x, y, z$ denote the equitable basis for the Lie algebra $s l_{2}$. Let $V_{d}$ denote a finite-dimensional irreducible $s l_{2}$-module, let $\left\{v_{i}\right\}_{i=0}^{d}$ be a basis of $V_{d}$ as in Lemma 3, let $e=\left\{t^{i} v_{i}\right\}_{i=0}^{d}$, and let $A \in \operatorname{End}\left(V_{d}\right)$ such that $A$ acts on e as in Lemma 16. Then, each of the pairs $[A]_{e},[x]_{e},[A]_{e},[y]_{e},[A]_{e},[z]_{e}$ is a Leonard pair of Krawtchouk type if and only if $r_{z} \neq 0, h \neq 0, t \neq 1, r_{z}+$ $2 h \neq 0$ and $r_{z}-t\left(r_{z}+2 h\right) \neq 0$.

Proof. The result holds from Lemmas 17, 22, and 23.

\section{A Basis of $A$}

Let $U\left(s l_{2}\right)$ denote the universal enveloping algebra of $s l_{2}$. Thus, $U\left(s l_{2}\right)$ is the associative $\mathscr{F}$-algebra with generators $x$, $y, z$ and relations $[x, y]=2 x+2 y,[y, z]=2 y+2 z$, and $[z, x]=2 z+2 x$, where $[n, m]=n m-m n$ is commutator of $n$ and $m$. In this section, we write $A$ as a linear combination of the generators of $U\left(s l_{2}\right)[16,20-23]$.

Definition 7. Let $Y \in U\left(s l_{2}\right)$ denote any linear combination of $1, x, y$, and $z$, and write

$$
Y=a_{I} I+a_{x} x+a_{y} y+a_{z} z
$$

where $a_{I}, a_{x}, a_{y}, a_{z} \in \mathscr{F}$.

Lemma 26. With reference to Lemma 3 and Definition 7 , let $t$ be a nonzero scalar in $\mathscr{F}$ and let $e_{i}=t^{i} v_{i}(0 \leq i \leq d)$; then,

$$
\begin{aligned}
Y e_{0} & =\kappa_{0} e_{0}+\eta_{1} e_{1} \\
Y e_{d} & =\mu_{d-1} e_{d-1}+\kappa_{d} e_{d} \\
Y e_{i} & =\mu_{i-1} e_{i-1}+\kappa_{i} e_{i}+\eta_{i+1} e_{i+1}, \quad(1 \leq i \leq d-1)
\end{aligned}
$$

where 


$$
\begin{aligned}
& \kappa_{i}=a_{I}+(d-2 i)\left(a_{z}-a_{x}-a_{y}\right), \quad(0 \leq i \leq d), \\
& \mu_{i}=2(d-i) t a_{x}, \quad(0 \leq i \leq d-1), \\
& \eta_{i}=\frac{-2 i a_{y}}{t}, \quad(1 \leq i \leq d) .
\end{aligned}
$$

Proof. Expand the action of $x, y$, and $z$ on $e=\left\{e_{i}\right\}_{i=0}^{d}$ using Lemma 8.

Lemma 27. Fix an integer $d \geq 3$; let $x, y, z$ denote the generators of $U\left(s l_{2}\right)$, let $A \in U\left(s l_{2}\right)$ such that $A=a_{I} I+a_{x} x+a_{y} y+a_{z} z$, let $V_{d}$ denote a finite-dimensional irreducible sl $l_{2}$-module, and let $e=\left\{t^{i} v_{i}\right\}_{i=0}^{d}$ be a basis of $V_{d}$, assume that $A$ acts on $e$ as in Lemma 16. Then,

$$
\begin{aligned}
& a_{I}=\theta_{0}+\frac{d h}{2}, \\
& a_{x}=\frac{r_{z}}{4 t}, \\
& a_{y}=\frac{t\left(r_{z}+2 h\right)}{4}, \\
& a_{z}=\frac{t\left(r_{z}+2 h\right)}{4}-\frac{r_{z}+h}{2}+\frac{r_{z}}{4 t}, \\
& r_{z}=4 t a_{x}, \\
& h=\frac{2}{t}\left(a_{y}-t^{2} a_{x}\right) .
\end{aligned}
$$

Proof. Compare the action of $A$ on $e$ with the action of $Y$ in Lemma 26 to get the result.

Lemma 28. With reference to Lemma 27, $a_{z}=t(1-t)\left(t a_{x}-a_{y}\right)$.

Proof. Routine calculations show that

$$
t(1-t)\left(t a_{x}-a_{y}\right)=\frac{t\left(r_{z}+2 h\right)}{4}-\frac{r_{z}+h}{2}+\frac{r_{z}}{4 t} .
$$

Lemma 29. Fix an integer $d \geq 3$; let $x, y, z$ denote the generators of $U\left(s l_{2}\right)$, let $A \in U\left(s l_{2}\right)$ such that $A=a_{I} I+a_{x} x+a_{y} y+a_{z} z$, where $a_{I}, a_{x}, a_{y}$, and $a_{z}$ in $\mathscr{F}$, and let $V_{d}$ denote a finite-dimensional irreducible $s_{2}$-module. Then, each of the pairs $A, x, A, y$, and $A, z$ is a Leonard pair of Krawtchouk type if and only if $a_{x}, a_{y}, a_{z}$ are nonzero scalars in $\mathscr{F}$ and there exists a nonzero scalar $t \in \mathscr{F}$ such that $a_{z}=$ $t(1-t)\left(t a_{x}-a_{y}\right)$ and $a_{y}-t^{2} a_{x} \neq 0$.

Proof. Assume all the pairs $A, x, A, y$, and $A, z$ are Leonard pairs of Krawtchouk type; then, by Lemma 25, there exist nonzero scalars $r_{z}, h, t \in \mathscr{F}, t \neq 1$ such that $A$ acts on the basis $\left\{t^{i} v_{i}\right\}_{i=0}^{d}$ as in Lemma 16. Moreover, $r_{z}+2 h \neq 0$ and $r_{z}-t\left(r_{z}+2 h\right) \neq 0$. Hence, $A$ acts on $\left\{t^{i} v_{i}\right\}_{i=0}^{d}$ as
$a_{I} I+a_{x} x+a_{y} y+a_{z} z$, where $a_{I}, a_{x}, a_{y}$, and $a_{z}$ are as in Lemma 27, and $a_{z}=t(1-t)\left(t a_{x}-a_{y}\right)$.

Note that $r_{z}, r_{z}+2 h$, and $h$ are nonzero scalars if and only if $a_{x}, a_{y}$, and $a_{y}-t^{2} a_{x}$ are nonzero scalars, respectively, and since $r_{z}-t\left(r_{z}+2 h\right)=4\left(t a_{x}-a_{y}\right)$, then, by Lemma 28, $r_{z}-t\left(r_{z}+2 h\right) \neq 0$ if and only if $a_{z} \neq 0$.

In the another direction, if $A=a_{I} I+a_{x} x+a_{y} y+a_{z} z$ for $a_{I} \in \mathscr{F}, a_{x}, a_{y}, a_{z}$ are nonzero scalars in $\mathscr{F}, a_{z}=t(1-$ t) $\left(t a_{x}-a_{y}\right)$ and $a_{y}-t^{2} a_{x} \neq 0$ for nonzero scalar $t \in \mathscr{F}$, then $a_{z} \neq 0$ implies $t \neq 1$; hence, we can find $r_{z}=4 t a_{x}$, $h=(2 / t)\left(a_{y}-t^{2} a_{x}\right)$ nonzero scalars such that $A$ acts on $\left\{t^{i} v_{i}\right\}_{i=0}^{d}$ as in Lemma 16 . Hence, the pairs $A, x, A, y$, and $A, z$ are Leonard pairs of Krawtchouk type by Lemma 25.

Proof of Theorem 10. Note that, from Lemma 4, $[x]_{u}=[y]_{w}=[z]_{v}$. Hence, the result holds from Lemmas 16,17 , and 24 .

Proof of Theorem 11. Clear from Lemmas 12, 25, and 29.

\section{Data Availability}

The data used to support the findings of this study are included within the article.

\section{Conflicts of Interest}

The authors declare that they have no conflicts of interest.

\section{References}

[1] P. Terwilliger, "The subconstituent algebra of an association scheme. III," Journal of Algebraic Combinatorics, vol. 2, no. 2, pp. 177-210, 1993.

[2] E. Bannai and T. Ito, Algebraic Combinatorics I, BenjaminCummings, Menlo Park, CA, USA, 1984.

[3] D. A. Leonard, "Orthogonal polynomials, duality and association schemes," SIAM Journal on Mathematical Analysis, vol. 13, no. 4, pp. 656-663, 1982.

[4] D. A. Leonard, "Parameters of association schemes that are both P- and Q-polynomial," Journal of Combinatorial Theory, Series A, vol. 36, no. 3, pp. 355-363, 1984.

[5] H. Alnajjar and B. Curtin, "Leonard pairs from the equitable basis of $s l_{2}$," The Electronic Journal of Linear Algebra, vol. 20, pp. 490-505, 2010.

[6] P. Terwilliger, "Introduction to leonard pairs. OPSFA Rome 2001," Journal of Computational and Applied Mathematics, vol. 153, no. 2, pp. 463-475, 2003.

[7] H. Alnajjar, "Leonard pairs associated with the equitable generators of the quantum algebra $U_{q}(\mathrm{sl} 2)$," Linear and Multilinear Algebra, vol. 59, no. 10, pp. 1127-1142, 2011.

[8] B. Curtin, "Modular leonard triples," Linear Algebra and its Applications, vol. 424, no. 2-3, pp. 510-539, 2007.

[9] B. Hartwig, "Three mutually adjacent Leonard pairs," Linear Algebra and its Applications, vol. 408, pp. 19-39, 2005.

[10] T. Ito and P. Terwilliger, "Finite-dimensional irreducible modules for the three-point 2 loop algebra," Communications in Algebra, vol. 36, no. 12, pp. 4557-4598, 2008.

[11] C. Kassel, Quantum Groups, Springer-Verlag, New York, NY, USA, 1995. 
[12] M. Sang, S. Gao, and B. Hou, "Leonard pairs and quantum algebra $U\left(s l_{2}\right)$," Linear Algebra and its Applications, vol. 510, pp. 346-360, 2016.

[13] K. Nomura, "Leonard pairs having LB-TD form," Linear Algebra and its Applications, vol. 455, no. 1-21, 2014.

[14] K. Nomura and P. Terwilliger, "Affine transformations of a Leonard pair," The Electronic Journal of Linear Algebra, vol. 16, pp. 389-418, 2007.

[15] P. Terwilliger, "Two linear transformations each tridiagonal with respect to an eigenbasis of the other; the TD-D canonical form and the LB-UB canonical form," Journal of Algebra, vol. 291, no. 1, pp. 1-45, 2005.

[16] P. Terwilliger, "Two linear transformations each tridiagonal with respect to an eigenbasis of the other," Linear Algebra and its Applications, vol. 330, no. 1-3, pp. 149-203, 2001.

[17] P. Terwilliger and R. Vidunas, "Leonard pairs and the AskeyWilson relations," Journal of Algebra and Its Applications, vol. 3, pp. 1-16, 2004.

[18] P. Terwilliger, "Two linear transformations each tridiagonal with respect to an eigenbasis of the other; comments on the parameter array," Designs, Codes and Cryptography, vol. 34, no. 2-3, pp. 307-332, 2005

[19] H. Alnajjar and B. Curtin, "A bidiagonal and tridiagonal linear map with respect to eigenbases of equitable basis of $s l_{2}$," Linear and Multilinear Algebra, vol. 61, no. 12, pp. 1668-1674, 2013.

[20] G. Benkart and P. Terwilliger, "The equitable basis for $s l_{2}$," Mathematische Zeitschrift, vol. 268, no. 1-2, pp. 535-557, 2011.

[21] P. Terwilliger, "Leonard pairs from 24 points of view," Rocky Mountain Journal of Mathematics, vol. 32, no. 2, pp. 827-888, 2002.

[22] P. Terwilliger, "Leonard pairs and the q-Racah polynomials," Linear Algebra and its Applications, vol. 387, pp. 235-276, 2004.

[23] P. Terwilliger, "An algebraic approach to the Askey scheme of orthogonal polynomials, orthogonal polynomials and special functions," in Lecture Notes in Math, Springer, Berlin, Germany, 2006. 